\title{
EVALUACIÓN DE MÉTODOS NO PARAMÉTRICOS PARA LA ESTIMACIÓN DE RIQUEZA DE ESPECIES DE PLANTAS LEÑOSAS EN CAFETALES
}

\author{
Ana María LÓPEZ-GÓmeZ y GuadALUPe WilLIAMS-LINERA \\ Departamento de Ecología Funcional, Instituto de Ecología, A.C., km 2.5 Carretera Antigua a Coatepec \\ No. 351, Congregación El Haya, Xalapa 91070, Veracruz, México. \\ Tel (228) 842-1800 ext. 4206, Fax (228) 842-1800 ext. 4222 ó 818-78-09. \\ Correo-e: amalgo@yahoo.com; lupew@ecologia.edu.mx
}

\begin{abstract}
Resumen: Los cafetales, como parte del paisaje, contribuyen a la diversidad regional de especies leñosas. La riqueza de árboles fue evaluada en ocho fincas cafetaleras activas y abandonadas, y se comparó la riqueza observada o muestreada con la riqueza estimada utilizando estimadores no paramétricos (ACE, ICE, Chao 1, Chao 2, Jackknife 1, Jackknife 2, Bootstrap) y modelos asintóticos (Clench, Lineal). La precisión de los estimadores se estimó evaluando el sesgo y la exactitud por medio de la comparación de la riqueza estimada con la riqueza verdadera o total. La riqueza observada en cafetales activos y abandonados fue de 55 y 61 especies de árboles, respectivamente; la mayoría de las especies tuvieron una distribución espacial agregada. Ninguna curva de acumulación de especies fue asintótica. El mejor comportamiento lo presentaron ICE y Chao 2. Los modelos paramétricos fueron los menos precisos y Jackknife 2 fue el mejor. Aunque con inconvenientes, los estimadores no paramétricos podrían ser los más adecuados para estimar la riqueza en sistemas manejados como cafetales.

Palabras clave: biodiversidad, cafetales de sombra, curvas de acumulación de especies, estimadores de riqueza, plantas leñosas.

Abstract: Shade-grown coffee plantations are part of the regional landscape and may contribute to the diversity of woody plants. Tree species richness was evaluated in eight active and abandoned coffee plantations. We used non-parametric (ACE, ICE, Chao 1, Chao 2, Jackknife 1, Jackknife 2, Bootstrap) and asymptotic models (Clench, Linear) to compare observed or sampled richness with estimated richness. The precision of the estimators was evaluated as bias and accuracy through the comparison of estimated richness and true (or total) richness values. Observed richness was 55 tree species in active coffee plantations and 61 species in abandoned plantations. Most species showed a clumped distribution. In no case did species-accumulation curves reach an asymptote. ICE and Chao 2 displayed the best performance. Parametric models were the least precise and Jackknife 2 was the most precise. Non-parametric estimators have disadvantages, but they appear to be appropriate to estimate richness in managed systems such as coffee plantations.
\end{abstract}

Key words: biodiversity, richness estimators, shade-grown coffee plantations, species-accumulation curves, trees.

$\mathbf{L}$ a riqueza, entendida como el número de especies, es la medida de biodiversidad empleada con mayor frecuencia (Gaston, 1996). Sin embargo, la medición de la riqueza en regiones extensas o con taxa diversos requiere de mucha inversión en esfuerzo de muestreo para obtener inventarios completos. Por lo tanto, se han desarrollado métodos de estimación de la riqueza a través de métodos de sustitución utilizando grupos indicadores o métodos de muestreo (Gaston, 1996; Gotelli y Colwell, 2001). Los métodos de estimación basados en muestras pueden ser por extrapolación a partir de curvas de acumulación de especies, o por uso de estimadores paramétricos y estimadores no paramétricos.

El método de extrapolación usa la curva observada de acumulación de especies para modelar el conteo de nuevas especies con respecto al esfuerzo de muestreo, y el valor de la riqueza es la asíntota de la curva (e.g. modelos de Clench o de Dependencia Lineal) (Palmer, 1990; Soberón y Llorente, 1993). Una desventaja de utilizar extrapolaciones es que se requieren datos de comunidades bien muestreadas para la predicción del valor asintótico (Hughes et al., 2001). La estimación paramétrica consiste en ajustar los 
datos de abundancia relativa de las especies en una muestra a un modelo matemático paramétrico como la serie logarítmica, la distribución log-normal, o el modelo de la vara quebrada (ver revisión en Moreno, 2001). Algunos impedimentos para usar métodos paramétricos es que siempre se requieren datos de abundancia relativa de las especies y que se deben hacer suposiciones sobre la verdadera distribución de abundancias en una comunidad (Hughes et al., 2001).

La estimación de la riqueza utilizando métodos no paramétricos proviene de la adaptación de los métodos de captura-recaptura, ya que la probabilidad de captura varía entre individuos en una población, así como la abundancia de las especies varía en un ensamble de especies. Los estimadores no paramétricos utilizan datos de presencia-ausencia o datos de abundancia de especies y se enfocan en las especies poco abundantes o raras, o sea las que se presentan solamente en una o dos muestras, o que tienen uno o dos individuos en el conjunto de muestras (Colwell y Coddington, 1994; Moreno, 2001). Entre las ventajas del uso de los métodos no paramétricos está que estos estimadores tienen un sesgo menor que la extrapolación basada en una curva de acumulación de especies (Colwell y Coddington, 1994; Gotelli y Colwell, 2001) y que requieren menor cantidad de datos que los métodos paramétricos (Brose, 2002). Algunos de los estimadores no paramétricos que se han desarrollado son Bootstrap, Jackknife, Chao, ACE, ICE y han sido revisados por Colwell y Coddington (1994) y Chazdon et al. (1998).

Al utilizar un estimador no paramétrico se debe evaluar su comportamiento ya que su eficacia puede ser diferente según la riqueza y complejidad del sistema, la proporción del área muestreada y el método de muestreo empleado. Se han propuesto varios criterios para seleccionar un estimador. Por ejemplo, un buen estimador debe ser insensible al tamaño de muestra, es decir, su curva de acumulación de especies debe tener una tasa de crecimiento inicial alta hasta llegar a una riqueza máxima y a la asíntota; también debe ser insensible al orden de muestreo y a la distribución espacial de las especies (Chazdon et al., 1998; Gotelli y Colwell, 2001). Además, el estimador debe ser eficaz en términos de sesgo (poco sesgado), exactitud y precisión (valor de estimación cercano al valor de la riqueza verdadera) (Palmer, 1990; Chiarucci et al., 2003).

La riqueza de especies en agroecosistemas ha sido estimada comúnmente utilizando índices de diversidad (Wunderle y Latta, 1996; Rojas et al., 2001; Decaëns y Jiménez, 2002). En algunas ocasiones se reporta el uso de la extrapolación en curvas de modelos asintóticos (Bandeira et al., 2005) o el ajuste a una distribución paramétrica (Perfecto y Vandermeer, 2002). La estimación no paramétrica ha sido poco utilizada para analizar la diversidad en agroecosistemas (Brose, 2002; Perfecto et al., 2003) y la evaluación del uso de los estimadores de riqueza en sistemas manejados se ha limitado a plantas en bosques de extracción (Skov y Lawesson, 2000) y escarabajos en un paisaje agrícola (Brose, 2002). En agroecosistemas como los cafetales de sombra tradicionales, la distribución y la riqueza de especies están influidas por un factor antropogénico, el cual posiblemente induzca a que los estimadores de riqueza tengan un comportamiento errático o diferente del que manifiestan en sistemas naturales.

Los cafetales forman una parte importante del paisaje en el centro de Veracruz. La distribución del cultivo de café en esta región coincide con la del bosque mesófilo de montaña o bosque de niebla (Challenger, 1998). La diversidad de especies de árboles en el bosque regional es alta, lo cual se debe a la diversidad beta o recambio de especies entre los fragmentos de bosque (Williams-Linera, 2002). Los pocos estudios sobre la diversidad de plantas leñosas en cafetales de la zona indican que la riqueza de las fincas individuales es baja (Hernández-Córdoba, 1979; Escamilla et al., 1994), así que consideramos que la diversidad beta podría llegar a ser alta como en el caso de los fragmentos de bosque regionales. Para conservar la biodiversidad en el ámbito regional sería importante incluir en programas de conservación, además de fragmentos del bosque original, otros usos tradicionales del suelo, como las fincas cafetaleras. Pero para evaluar la contribución de los cafetales a la diversidad regional, primero se deben tener estimaciones confiables de la diversidad alfa, pues a partir de ella se evalúa la diversidad beta y gamma (Magurran, 2004).

El objetivo del presente estudio fue evaluar la aplicación de diferentes métodos de estimación de la riqueza de especies en el análisis de la biodiversidad de cafetales de sombra activos y abandonados.

\section{Materiales y métodos}

Inventario. El área de estudio se ubica en los alrededores de Xalapa, Veracruz, México $\left(19^{\circ} 25^{\prime}\right.$ - $19^{\circ} 35^{\prime}$ N y 965' $96^{\circ} 58^{\prime} \mathrm{O}$ ) entre 1,136 y $1,483 \mathrm{~m}$ de altura. El tipo de vegetación característico es el bosque mesófilo de montaña. Algunas especies dominantes de árboles son Carpinus caroliniana Walter, Clethra mexicana DC., Liquidambar styraciflua L. var. mexicana Oersted, Quercus acutifolia Née, $Q$. germana Schltdl. et Cham., Q. xalapensis Bonpl. y Turpinia insignis (Kunth) Tul. En esta región se seleccionaron cuatro cafetales de sombra activos y cuatro cafetales abandonados $(10-20$ años) que tuvieron un manejo de policultivo. En cada sitio de estudio se identificaron especies de plantas leñosas $5 \mathrm{~cm}$ diámetr o a $1.3 \mathrm{~m}$ (DAP) y se contaron el número de individuos por especie en diez lotes de $20 \times 20 \mathrm{~m}$ en cafetales activos y diez lotes de muestreo de $10 \times 10 \mathrm{~m}$ en cafetales abandonados (detalles en López-Gómez, 2004).

La riqueza de especies en ambos tipos de cafetal se 
analizó utilizando siete estimadores no paramétricos (ACE, ICE, Chao 1, Chao 2, Jackknife 1, Jackknife 2 y Bootstrap) mediante el programa EstimateS versión 7 (Colwell, 2004), con las opciones preestablecidas en el programa. El esfuerzo de muestreo en este estudio fue el número de lotes muestreado y se graficó en el eje de las abcisas de las curvas de acumulación de especies (Gotelli y Colwell, 2001).

Distribución espacial. Para evaluar si las especies presentan una distribución agregada en las muestras, se trazó la curva de Coleman para cada uso del suelo. Esta curva aleatoriza la posición de los individuos en las muestras. Por lo tanto, si existen diferencias entre la curva de acumulación de todos los individuos observados y la curva de Coleman significa que las especies raras están agrupadas en pocas muestras. Al contrario, una mayor sobreposición de la curva de acumulación de especies observada y la curva de Coleman señala una distribución más aleatoria de las especies (Colwell y Coddington, 1994).

Pa ra determinar el patrón de distribución de los individuos de cada especie se usó el llamado método de cociente varianza/media. Este método presupone una distribución Poisson y consiste en comparar el número de individuos en los lotes y los resultados esperados si la muestra tuviera una distribución al azar. El patrón de distribución es al azar si el cociente varianza/media es 1 , es uniforme si el cociente es $<1$ y el patrón se considera agrupado si el cociente es $>1$ (Rabinovich, 1980).

Evaluación de los estimadores. El comportamiento de las curvas de acumulación de especies de los estimadores no paramétricos se analizó visualmente utilizando todos los datos de cada tipo de cafetal. Se consideró que los estimadores de riqueza más adecuados serían aquellos cuyas curvas de acumulación tuvieran una tasa de crecimiento inicial alta y una asíntota definida (Chazdon et al., 1998; Gotelli y Colwell, 2001).

Todos los estimadores no paramétricos se evaluaron en términos de sesgo y exactitud, y además se incluyeron dos estimadores asintóticos (modelo de Clench y modelo de Dependencia Lineal). Para llevar a cabo la evaluación es necesario tener una medida de la riqueza verdadera (Sverdadera) de plantas leñosas o el censo de la riqueza total en cada tipo de cafetal. Para esto se seleccionó un cafetal activo y un cafetal abandonado (los que tenían menor área), y en cada uno se contaron absolutamente todas las especies de árboles presentes en el área, tanto dentro como fuera de los lotes de muestreo. Mediante la relación de la riqueza estimada (Sestimada) y la riqueza verdadera ( S $_{\text {verdadera }}$ ) se calculó el sesgo y la exactitud de nueve estimadores de riqueza. Las fórmulas utilizadas fueron (Chiarucci et al., 2003):

$$
\begin{gathered}
\text { Sesgo }=\left(S_{\text {estimada }}-S_{\text {verdadera }}\right) / S_{\text {verdadera }} \\
\text { Exactitud }=\left(\left(S_{\text {estimada }}-S_{\text {verdadera }}\right) / S_{\text {verdadera }}\right)^{2}
\end{gathered}
$$

El sesgo indica la sobreestimación o la subestimación de la riqueza y la exactitud indica la cercanía de la riqueza estimada a la riqueza verdadera. Tanto el sesgo como la exactitud tienen valores de -1 a 1 , y los valores cercanos a cero son los menos sesgados o los más exactos, respectivamente (Palmer, 1990; Brose et al., 2003).

\section{Resultados}

Inventario. En total se registraron 84 especies de plantas leñosas $5 \mathrm{~cm}$ en las 80 unidades de $\mathrm{m}$ uestreo tomadas en cafetales activos y abandonados (López-Gómez, 2004). Cincuenta y cinco son especies nativas y de ésas, $51 \%$ son primarias. En los cuatro cafetales activos, el inventario fue de 55 especies, de las cuales $53 \%$ fueron especies nativas, mientras que en los cuatro cafetales abandonados se registraron 61 especies, $77 \%$ de ellas nativas (cuadro 1). En los dos tipos de cafetales se encontró un número similar de individuos (420 y 439), pero en cafetales activos se muestreó un área mayor.

El número de especies raras (aquellas que tuvieron entre 1 y 2.5 individuos/ha) fue mayor en los cafetales activos $(51 \%)$ que en los cafetales abandonados (28\%). En ambos tipos de cafetal, las especies únicas (presentes en un solo sitio) tuvieron un porcentaje $>50 \%$, y el porcentaje de especies con un solo individuo (singletons) fue semejante (27 y $26 \%$, cuadro 1 ).

Cuadro 1. Datos de riqueza de especies de árboles con DAP $5 \mathrm{~cm}$ en cafetales activos y abandonados del centro de Veracruz, México. Los datos que se presentan son el número de lotes muestreado $(\mathrm{N})$, la densidad de individuos/ha, la riqueza observada en las muestras (Sobservada), la riqueza observada de especies nativas (Snativas), especies raras sensu Gimaret-Carpentier et al. (1998), especies únicas o presentes en una sola unidad de muestreo, singletons o especies con un solo individuo, y la riqueza estimada por siete indicadores no paramétricos de diversidad. El valor de dispersión es \pm la desviación estándar.

\begin{tabular}{lcc}
\hline & $\begin{array}{c}\text { Cafetal } \\
\text { activo }\end{array}$ & $\begin{array}{c}\text { Cafetal } \\
\text { abandonado }\end{array}$ \\
\hline $\mathrm{N}$ & 40 & 40 \\
Densidad & $341 \pm 53$ & $1070 \pm 56$ \\
Sobservada & 55 & 61 \\
Snativas & 29 & 47 \\
Raras (1-2.5 individuos/ha) & 28 & 16 \\
Únicas & 33 & 35 \\
Singletons & 15 & 16 \\
ACE & $67 \pm 0$ & $74 \pm 0$ \\
ICE & $83 \pm 0.02$ & $81 \pm 0.01$ \\
Chao 1 & $64 \pm 6.9$ & $81 \pm 17.9$ \\
Chao 2 & $87 \pm 22.14$ & $82 \pm 15.1$ \\
Jackknife 1 & $77 \pm 6.23$ & $81 \pm 4.63$ \\
Jackknife 2 & $93 \pm 0$ & $92 \pm 0$ \\
Bootstrap & $65 \pm 0$ & $70 \pm 0$ \\
\hline
\end{tabular}


Distribución espacial. Las especies muestreadas en cafetales activos y abandonados presentaron una distribución espacial agregada, de acuerdo con las curvas de Coleman y con el método del cociente varianza/media. La curva de Coleman se encontró por arriba de la curva de acumulación de especies, indicando que las especies relativamente raras aparecieron en pocas muestras y que éstas están agregadas (figura 1a, b). El cociente varianza/media indicó que la dis- tribución espacial de la mayoría de las especies en cafetales fue principalmente agregada. Sin embargo, la mayoría de las especies no nativas fueron las que tuvieron distribución agrupada, mientras que la mayoría de las especies nativas estuvieron distribuidas al azar (cuadro 2).

Evaluación de los estimadores. Todos los estimadores no paramétricos de riqueza de especies se comportaron de

Cuadro 2. Distribución espacial según el método del cociente varianza/media de especies de plantas leñosas en cafetales activos y abandonados del centro de Veracruz, México.

\begin{tabular}{cccc}
\hline & $\begin{array}{c}\text { No. de especies } \\
\text { no nativas }\end{array}$ & $\begin{array}{c}\text { No. de especies } \\
\text { nativas }\end{array}$ & $\begin{array}{c}\text { No. total } \\
\text { de especies }\end{array}$ \\
\hline $\begin{array}{c}\text { Cafetales activos } \\
\text { Distribución agrupada }\end{array}$ & 16 & 8 & 24 \\
Distribución al azar & 9 & 21 & 30 \\
Cafetales abandonados & & & 21 \\
Distribución agrupada & 7 & 14 & 39 \\
Distribución al azar & 6 & 33 & 3 \\
\hline
\end{tabular}
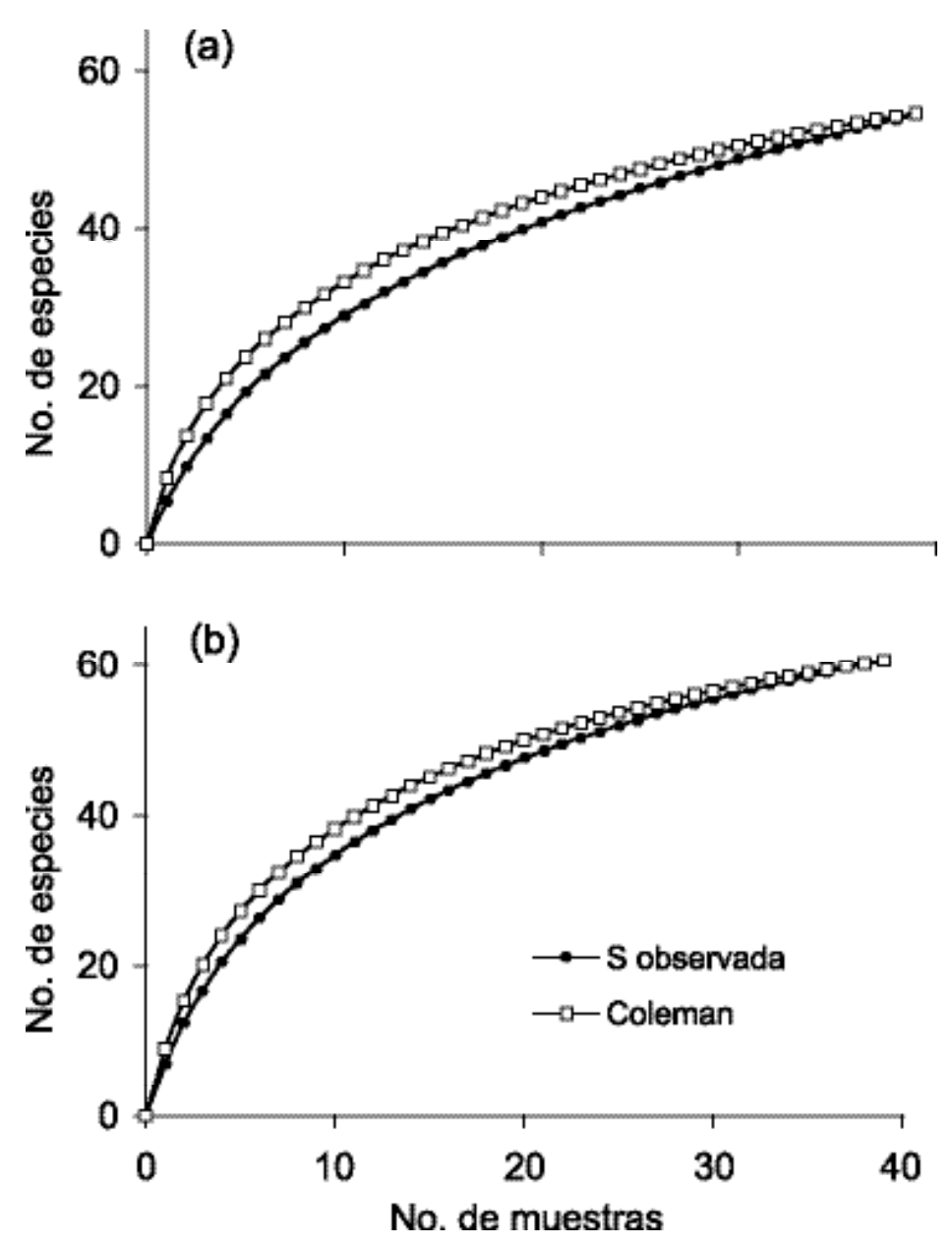

Figura 1. Curvas de Coleman para explorar el nivel de agregación de las especies en las muestras de (a) cafetales activos, y (b) cafetales abandonados en el centro de Veracruz, México. 

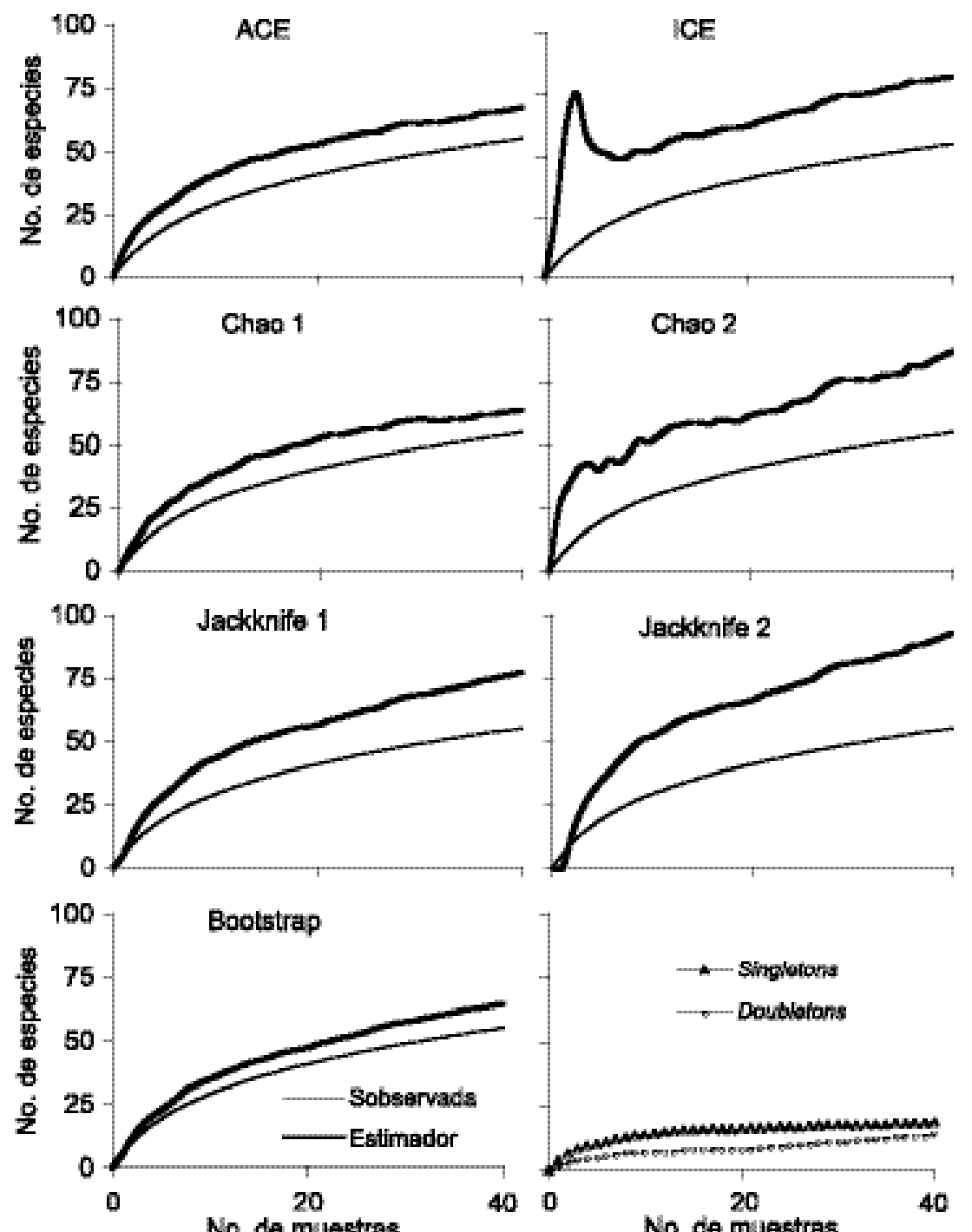

Figura 2. Curvas de acumulación de especies de la riqueza observada y la riqueza estimada por siete estimadores no paramétricos, y sin gletons y doubletons para plantas leñosas $5 \mathrm{~cm} \mathrm{D} \mathrm{AP} \mathrm{en} \mathrm{cafetales} \mathrm{activos.}$

manera diferente (figuras 2, 3, cuadro 1). Las curvas de acumulación de especies en los dos tipos de cafetal no alcanzaron una asíntota definida, y las curvas que tuvieron un crecimiento inicial alto fueron las de los estimadores ICE y Chao 2 (figuras 2, 3). Además, en ninguno de los dos tipos de cafetal la curva de singletons tendió a declinar o a sobreponerse con la de doubletons (figuras 2, 3). En los cafetales activos el intervalo de riqueza total estimada fue 64 - 93 especies de plantas leñosas, mientras que en cafetales abandonados dicho intervalo fue 70 - 92 especies. Cabe señalar que cuatro estimadores (ICE, Chao 1, Chao 2 y Jackknife 1) en los sitios abandonados coinciden en un valor de riqueza de 81 a 82 especies para todas las especies agrupadas (cuadro 1).

La evaluación de la precisión de los estimadores mostró que el cafetal activo tuvo una riqueza muestreada de 22 especies y una riqueza verdadera de 44 especies, mientras que en el cafetal abandonado la riqueza muestreada fue de 27 especies, pero la verdadera alcanzó 47 especies (cuadro 3). La evaluación de la eficacia de los estimadores con respecto a la riqueza real mostró que el estimador que tuvo el menor sesgo y la mayor exactitud en el cafetal activo fue el Jackknife 2, seguido por ICE, Jackknife 1, Chao 2, Chao 1, ACE y finalmente Bootstrap. En el cafetal abandonado también fue Jackknife 2 el estimador menos sesgado y el más exacto, seguido por Jackknife1, ICE, Chao 2, ACE, Bootstrap y por último Chao1. Los estimadores no paramétricos de la riqueza verdadera más exactos fueron también los que más sobreestimaron la riqueza observada (cuadro 3). Los modelos de Clench y de Dependencia 
Ana María LóPeZ-GómeZ y GuAdALuPe Williams-LinERA
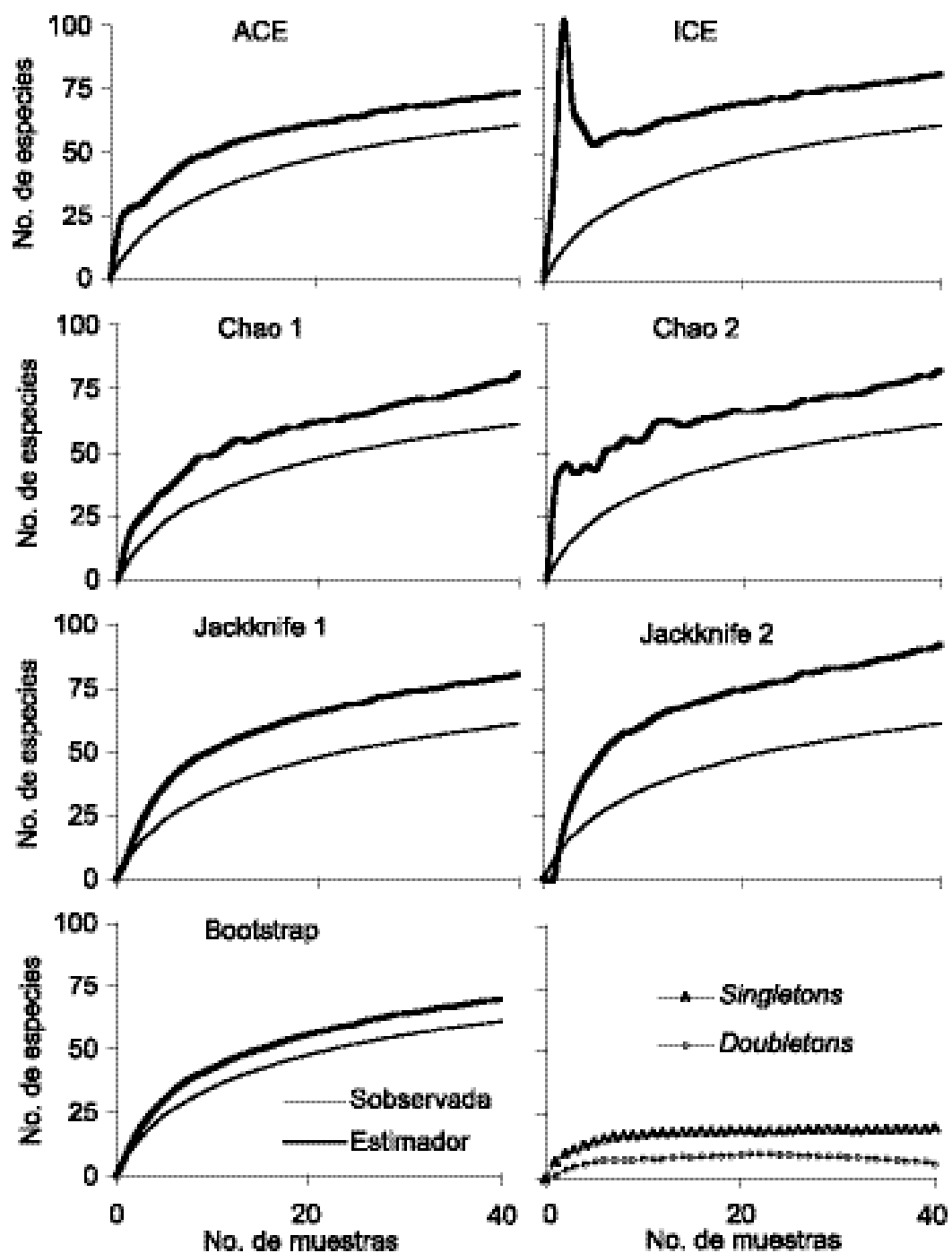

Figura 3. Curvas de acumulación de especies de la riqueza observada y la riqueza estimada por siete estimadores no paramétricos, y sin gletons y doubletons para plantas leñosas $5 \mathrm{~cm}$ D AP en cafetales abandonados del centro de Veracruz, México.

Cuadro 3. Riqueza observada (Sobservada), riqueza verdadera (Sverdadera) y riqueza estimada (Sestimada) y su sesgo y exactitud, para especies de plantas leñosas $5 \mathrm{~cm}$ en un cafetal activo y un cafetal abandonado en el centro de Veracruz, México.

\begin{tabular}{|c|c|c|c|c|c|c|c|c|c|c|}
\hline & & \multicolumn{7}{|c|}{ Estimadores no paraméticos } & \multicolumn{2}{|c|}{ Modelos asintóticos } \\
\hline & & ACE & ICE & Chao 1 & Chao 2 & Jackknife & Jackknife 2 & Bootstrap & Clench & Lineal \\
\hline \multicolumn{11}{|c|}{ Cafetal activo } \\
\hline Sobservada & 22 & & & & & & & & & \\
\hline Sverdadera & 44 & & & & & & & & & \\
\hline Sestimada & & 28 & 37 & 28 & 31 & 32 & 37 & 26 & 67 & 54 \\
\hline Sesgo & & -0.355 & -0.159 & -0.369 & -0.295 & -0.275 & -0.156 & -0.400 & 0.520 & 0.230 \\
\hline Exactitud & & 0.126 & 0.025 & 0.136 & 0.087 & 0.076 & 0.024 & 0.160 & 0.270 & 0.050 \\
\hline \multicolumn{11}{|c|}{ Cafetal abandonado } \\
\hline Sobservada & 27 & & & & & & & & & \\
\hline Sverdadera & 43 & & & & & & & & & \\
\hline Sestimada & & 31 & 34 & 29 & 32 & 35 & 37 & 31 & 67 & 54 \\
\hline Sesgo & & -0.271 & -0.209 & -0.333 & -0.256 & -0.184 & -0.132 & -0.280 & 0.560 & 0.260 \\
\hline Exactitud & & 0.074 & 0.044 & 0.111 & 0.065 & 0.034 & 0.017 & 0.078 & 0.310 & 0.070 \\
\hline
\end{tabular}


Lineal sobreestimaron entre 30 y $50 \%$ la riqueza verdadera del cafetal activo y del abandonado (cuadro 3).

\section{Discusión}

La riqueza observada a partir de muestreos (Sobservada) es una subestimación de la riqueza verdadera, pues siempre hay especies que no se registran en el inventario. Entonces es necesario estimar la riqueza verdadera a partir de esa riqueza muestreada mediante algún método estadístico (Palmer, 1990; Chiarucci et al., 2003). En varios estudios se ha llevado a cabo la evaluación visual de los estimadores a través de las curvas de acumulación de especies, y en ellos se han llegado a diferentes conclusiones (Chazdon et al., 1998; Gimaret-Carpentier et al., 1998; Skov y Lawesson, 2000; Longino et al., 2002). Chazdon et al. (1998) evaluaron la riqueza de plántulas e individuos juveniles de especies leñosas en seis sitios de selva primaria y secundaria, comparando el comportamiento de la curva de acumulación de especies de Chao 1 y 2, ACE, ICE, Jackknife 1 y 2, Bootstrap y Michaelis-Menten. Estos autores encontraron que los estimadores ICE y Chao 2 son los mejores estimadores de riqueza, ya que presentaron curvas de acumulación con un crecimiento inicial rápido y alcanzaron una asíntota definida. También concluyeron que a través de las unidades de muestreo, la tasa inicial de acumulación de especies en un muestreo es mayor si las especies tienen una distribución al azar en lugar de presentar una distribución agregada. Por lo tanto, dichos autores reportaron que el comportamiento de los estimadores no paramétricos se relaciona con la sensibilidad que tengan a la agregación; por ejemplo, Chao 1 y ACE son muy sensibles a la agre gación y Chao 2 e ICE lo son menos. Posiblemente la distribución no azarosa de las especies en los cafetales influyó en el funcionamiento de los estimadores no paramétricos, pues se encontró que solamente las curvas de acumulación de especies de los estimadores ICE y Chao 2 (estimadores poco sensibles a la agregación) mostraron una tasa de crecimiento inicial alta.

Por otra parte, Gimaret-Carpentier et al. (1998), utilizando datos de riqueza de árboles de dos bosques tropicales primarios para evaluar los estimadores Chao y Jackknife, encontraron que la proporción de especies raras presentes en la muestra influye en la forma de la curva de estimación. En el sitio con alto nivel de rareza (90\% de especies con 1 ó 2 individuos/ha) ninguna de las curvas alcanzó una asíntota, mientras que en el sitio con menor nivel de rareza (65\% de especies con 1 ó 2 individuos/ha), las curvas de Chao 1, Chao 2 y Jackknife 2 alcanzaron una asíntota. En los cafetales, la rareza medida de esa manera no fue tan alta, pero hubo más especies raras en los cafetales activos que en los abandonados y quizá ello influyó en el comportamiento poco claro de los estimadores en los cafetales activos. El número de singletons es diferente de cero y ninguna curva de acumulación de especies alcanzó una asíntota definida en los dos tipos de cafetal. Esto posiblemente se debe a que el cafetal es un sistema muy heterogéneo, según reportan Bandeira et al. (2005), y por lo tanto se necesitaría un número mucho más grande de unidades de muestreo para completar el inventario.

En los cafetales activos se observó una distribución relativamente más agregada que en los cafetales abandonados. Este patrón se relaciona con el hecho de que en los sitios activos hubo un mayor porcentaje de especies únicas y una densidad de especies menor que en los abandonados. La distribución espacial de las especies tiene consecuencias importantes para la estimación de la riqueza de especies (Palmer y White, 1994). En el caso de los estimadores no paramétricos, su comportamiento se puede modificar dependiendo de si las especies están o no distribuidas al azar (Chazdon et al., 1998). Se considera que cuando aumenta la agregación también aumenta la presencia de las especies en una sola muestra (Magurran, 2004) y que el grado de agregación está relacionado negativamente con la densidad de las especies (Condit et al., 2000). En los fragmentos de bosque de niebla de la región, aparentemente hay una tendencia a que la distribución de muchas especies arbóreas sea al azar (G. Williams-Linera, datos no publicados). Al parecer, la intervención humana y el manejo en los cafetales han modificado la composición y la distribución espacial de las especies en tal magnitud que la distribución azarosa del bosque original ha cambiado hacia una distribución agregada.

La evaluación del sesgo y la exactitud de los estimadores de riqueza han sido reportadas para datos reales de distintos grupos biológicos (Coddington et al., 1996; Palmer, 1990, 1991; Walther y Morand, 1998; Hellman y Fowler, 1999; Brose, 2002; Chiarucci et al., 2003). Los estudios que se han enfocado en las plantas leñosas señalan a los estimadores Jackknife como los más adecuados, en coincidencia con el presente estudio. Palmer $(1990,1991)$ utilizó datos de las especies de plantas de 30 lotes de 0.1 ha y comparó estimadores paramétricos (logarítmicos, Monod y Preston) con no paramétricos (Jackknife 1, Jackknife 2 y Bootstrap), y encontró que Jackknife 1 era el menos sesgado y más preciso de todos. Hellman y Fowler (1999), con datos de plantas leñosas $>1.5 \mathrm{~cm}$ DAP en cinco lotes de 0.4 ha, evaluaron Jackknife 1 y 2 y Bootstrap, y concluyeron que con tamaños de muestra pequeños el estimador Jackknife 2 es el más exacto y el menos sesgado, pero que en muestras con tamaños grandes, es el Jackknife 1 el que presenta valores más cercanos a la riqueza verdadera y con menos sesgo. Chiarucci et al. (2003) utilizaron datos de plantas perennes $>5 \mathrm{~cm}$ DAP en dos lotes de 0.1 ha para evaluar los mismos estimadores que Hellman y Fowler (1999), incluyendo además al Chao 2, y llegaron a una conclusión semejante sobre la conducta de los estimadores Jackknife. 
En los cafetales, los modelos asintóticos de Clench y de Dependencia Lineal probaron ser los menos adecuados para estimar la riqueza de plantas. El modelo de Dependencia Lineal se recomienda para estudios en los que el taxón está bien conocido y el área de estudio es pequeña (Soberón y Llorente, 1993). A pesar de ello, en el presente estudio ese modelo no funcionó apropiadamente. Cabe señalar que Palmer $(1990,1991)$ comparó estimadores paramétricos con no paramétricos y encontró que los paramétricos presentaron mayor imprecisión en la estimación de riqueza. Sin embargo, en otros organismos como ranas, murciélagos y escarabajos, se observó un comportamiento eficaz de los modelos de Clench y de Dependencia Lineal en sistemas manejados de la región (Moreno y Halffter, 2000; Pineda et al., 2005).

El hecho de tener un valor de la riqueza verdadera de cada tipo de cafetal brinda la posibilidad de evaluar el sesgo y la exactitud de los estimadores. Sin embargo, cuando se tienen áreas mayores o grupos más diversos, obtener el valor de la riqueza verdadera es difícil y por lo tanto la evaluación de la precisión de los estimadores se imposibilita. Debido a esta dificultad, consideramos que es práctico recurrir a la evaluación visual de los estimadores de riqueza, identificando su eficacia a partir de las características de la curva de acumulación de especies. Aparentemente, tanto los modelos asintóticos como los no paramétricos son poco apropiados para la estimación de riqueza de plantas en sistemas no naturales como los cafetales. Sin embargo, aun con los inconvenientes que presentan, los estimadores no paramétricos prometen ser más adecuados en la estimación de riqueza de cafetales, ya que tienen una mejor aproximación y tienden a sobreestimar menos a la riqueza verdadera que los modelos asintóticos. Finalmente, recomendamos probar estos estimadores en otros sistemas modificados, ya sea en agroecosistemas, plantaciones o estados sucesionales, y corroborarlos con controles que pueden ser sistemas naturales poco modificados.

\section{Agradecimientos}

Agradecemos a María de Jesús Peralta por su ayuda en el trabajo de campo y a Federico Escobar y Klaus Mehltreter por sus valiosos comentarios. CONACYT otorgó la beca No. 164466 a A.M. López-Gómez para estudios de maestría en el Instituto de Ecología, A.C. Este trabajo fue financiado por BIOCORES, ICA4-CT-2001-10095, programa INCO de la CE.

\section{Literatura citada}

Bandeira F.P., Martorell C., Meave J.A. y Caballero J. 2005. The role of rustic coffee plantations in the conservation of wild tree diversity in the Chinantec region of México. Biodiversity and Conservation 14:1225-1240.

Brose U. 2002. Estimating species richness of pitfall catches by non-parametric estimators. Pedobiologia 46:101-107.

Brose U., Martínez N.D. y Williams R.V. 2003. Estimating species richness: sensitivity to sample coverage and insensitivity to spatial patterns. Ecology 84:2364-2377.

Challenger A. 1998. Utilización y Conservación de los Ecosistemas Terrestres de México. Pasado, Presente y Futuro. Comisión Nacional para el Conocimiento y Uso de la Biodiversidad, Instituto de Biología, UNAM y Agrupación Sierra Madre, S.C., México, D.F.

Chazdon R.L., Colwell R.K., Denslow J.S. y Guariguata M.R. 1998. Statistical methods for estimating species richness of woody regeneration in primary and secondary rain forest of northeastern Costa Rica. En: Dallmeier F. y Comiskey J.A. Eds. Forest Biodiversity Research, Monitoring and Modelling, pp. 285-309, The Parthenon Publishing Group, París.

Chiarucci A., Enright N.J., Perry G.L., Miller B.P. y Lamont B.B. 2003. Performance of nonparametric species richness estimators in a high dive rsity plant community. Diversity and Distributions 9:283-295.

Coddington J.A., Young L.H. y Coyle F.A. 1996. Estimating spider species richness in a southern Appalachian cove hardwood forest. The Journal of Arachnology 24:111-128.

Colwell R.K. 2004. EstimateS: Statistical Estimation of Species Richness and Shared Species for Samples. Version 7. Aplicación publicada en:

$<$ http://viceroy.eeb.uconn.edu/estimates >

Colwell R.K. y Coddington J.A. 1994. Estimating terrestrial biodiversity through extrapolation. Philosophical Transactions of the Royal Society, London (Series B) 345:101-118.

Condit R., Ashton P.S., Baker P., Bunyavejchewin S., Gunatilleke S., Gunatilleke N., Hubbell S.P., Foster R.B., Itoh A., LaFrankie J.V., Lee H.S., Losos E., Manokaran N., Sukumar R. y Yamakura T. 2000. Spatial patterns in the distribution of tropical tree species. Science 288:1414-1418.

Decaëns T. y Jiménez J.J. 2002. Earthworm communities under an agricultural intensification gradient in Colombia. Plant and Soil 240:133-143.

Escamilla E., Licona A., Díaz S., Cortés S., Sosa R. y Rodríguez L. 1994. Los sistemas de producción del café en el centro de Veracruz, México. Un análisis tecnológico. Revista de Historia (Centro de Investigaciones Históricas Universidad de Costa Rica) 30:41-67.

Gaston K.J. 1996. Biodiversity: A Biology of Numbers and Difference. Blackwell Science, Oxford.

Gimaret-Carpentier C., Pélissier R., Pascal J.P. y Houllier F. 1998. Sampling strategies for the assessment of tree species diversity. Journal of Vegetation Science 9:161-172.

Gotelli N.J. y Colwell R.K. 2001. Quantifying biodiversity: procedures and pitfalls in the measurement and comparison of species richness. Ecology Letters 4:379-391.

Hellmann J.J. y Fowler G.W. 1999. Bias, precision, and accuracy of four measures of species richness. Ecological Applications 9:824-834.

Hernández-Córdoba M. 1979. Identificación de las principales especies de árboles utilizadas como sombra del cafeto en la cuenca de Coatepec. Tesis de Licenciatura, Facultad de Biología, Universidad Veracruzana, Xalapa, Veracruz, 107 pp. 
Hughes J., Hellmann J., Ricketts T. y Bohannan B. 2001. Counting the uncountable: statistical approaches to estimating microbial diversity. Applied and Environmental Microbiology 67:4399-4406.

López-Gómez A.M. 2004. Los cafetales de sombra como reservorio de la biodiversidad de plantas leñosas del bosque mesófilo de montaña del centro de Veracruz. Tesis de Maestría, Instituto de Ecología, A.C., Xalapa, Veracruz, 81 pp.

Longino J., Coddington J.A. y Colwell R.K. 2002. The ant fauna of a tropical rain forest: estimating species richness in three different ways. Ecology 83:689-702.

Magurran A.E. 2004. Measuring Biological Diversity. Blackwell Publishing, Oxford.

Moreno C.E. 2001. Métodos para Medir la Biodiversidad. CYTED, ORCYT/UNESCO y SEA, Zaragoza.

Moreno C.E. y Halffter G. 2000. Assessing the completeness of bat biodiversity inventories using species accumulation curves. Journal of Applied Ecology 37:149-158.

Palmer M. 1990. The estimation of species richness by extrapolation. Ecology 71:1195-1198.

Palmer M. 1991. Estimating species richness: the second order jackknife reconsidered. Ecology 72:1512-1513.

Palmer M. y White P.S. 1994. Scale dependence and the speciesarea relationship. The American Naturalist 144:717-740.

Perfecto I. y Vandermeer J. 2002. Quality of agroecological matrix in a tropical montane landscape: ants in coffee plantations in southern México. Conservation Biology 16:174-182.

Perfecto I., Mas A., Dietsch T.V. y Vandermeer J. 2003.
Conservation of biodiversity in coffee agroecosystems: a tritaxa comparison in southern México. Biodiversity and Conservation 12:1239-1252.

Pineda E., Moreno C.E., Escobar F. y Halffter G. 2005. Frog, bat and dung beetle diversity in the cloud forest and coffee agroecosystems of Veracruz, Mexico. Conservation Biology 19:400-410.

Rabinovich J.E. 1980. Introducción a la Ecología de Poblaciones Animales. Compañía Editorial Continental S.A., México, D.F.

Rojas L., Godoy C., Hanson P., Kleinn C. y Hilje L. 2001. Hopper (Homoptera: Auchenorryncha) diversity in shaded coffee systems of Turrialba, Costa Rica. Agroforestry Systems 53:171177.

Skov F. y Lawesson J.E. 2000. Estimation of plant species richness from systematically placed plots in a managed forest ecosystem. Nordic Journal of Botany 20:477-483.

Soberón J. y Llorente J. 1993. The use of species accumulation functions for the prediction of species richness. Conservation Biology 7:480-488.

Walther B.A. y Morand S. 1998. Comparative performance of species estimation methods. Parasitology 116:395-405.

Williams-Linera G. 2002. Tree species richness complementarity, disturbance and fragmentation in a Mexican tropical montane cloud forest. Biodiversity and Conservation 11:1825-1843.

Wunderle J.M. y Latta S.C. 1996. Avian abundance in sun and shade coffee plantations and remnant pine forest in the Cordillera Central, Dominican Republic. Ornitologia Neotropical 7:19-34.

Fecha de recepción: 22 de junio de 2005

Versión corregida: 29 de marzo de 2006

Aceptado: 30 de marzo de 2006 